\title{
Le culte de Santa Librada à Sigüenza: Patronage urbain et emblématique impériale *
}

Santa Librada fut aux siècles passés l'un des grands recours des femmes stériles et des femmes en couches, qui attendaient qu'elle mît fin à l'angoisse d'une vie sans postérité ou à la douleur d'un accouchement difficile ${ }^{1}$. Mais elle n'était après tout que l'un de ces saints de la délivrance, comme san Domingo de Silos, san Ramon Nonnato, santa Casilda ou san Cayetano, qui permettaient aux couples stériles d'obtenir enfin la descendance tant désirée, et aux femmes de se libérer de l'enfant prisonnier de leur corps ${ }^{2}$. Ces saints sont à l'origine de quantité de miracles, consignés dans des recueils largement diffusés à partir du xvile siècle. Bien que cette dimension du culte soit passionnante, elle n'est pas au cœur de ce travail.

Librada est une sainte peu commune. Elle est née d'un part multiple, et son légendaire, comme son martyre d'ailleurs, pose bien des questions. Enrôlée par l'idéologie de la Reconquista, elle contribue à Sigüenza, au début du xvie siècle, à la définition d'un nouvelle image du souverain. Derrière le choix de cette sainte, "importée" d'Aquitaine au xiıe siècle, se cache donc une volonté politique. Ce sont ces aspects, insuffisamment mis en lumière jusqu'ici, qui seront l'objet principal de cette étude.

* Une première version de ce texte a été présenté au séminaire de Bernard VINCENT en 1989.

1 C'est sans doute pour cette raison que, dans le diocèse de Sigüenza, Santa Librada est quelquefois représentée enceinte. Antonio CASTILLO DE LUCAS, Historias y tradiciones de Guadalajara y su provincia, (Guadalajara, 1970); J. R. LÓPEZ DE LOS MOZOS, Notas de etnología y folklore de Guadalajara, (Guadalajara, 1979); Gran Enciclopedia de Madrid, Castilla-La Mancha, t. VI, pp. 1654-1655. En Galice et León, le vocable «librar" est synonyme d'accoucher; et les femmes enceintes l'invoquaient autrefois en répétant: "De nuestro parto Librada!" A propos du culte rendu à santa Librada comme patronne des femmes en couches, cf. Antonio CASTILLO DE LUCAS, "La popularidad de Santa Librada, Abogada Celestial de los partos difíciles. Ensayo de hagiografía legendaria a través de un pliego de gozos"; extrait de Práctica Médico-Quirúrgica Internacional, Diciembre 1961-Enero 1962. 5 pages.

2 Sur ces saints favorables au bon accouchement et à la bonne naissance, cf. Jacques GÉLIS, "Ouvrir ou fermer le corps. Saintes et saints de la délivrance dans l'Espagne du xvie et du xvile siècle», dans Le corps comme métaphore dans l'Espagne des XVIe et XVIIe siècles, publié par Augustin REDONDO (Paris: Sorbonne, 1992), pp. 163-179. 
Celle-ci s'appuiera en particulier sur l'iconographie, puisque ce sont des images de la sainte qui sont à l'origine de cette recherche.

\section{UN LÉGENDAIRE DU PART MULTIPLE}

La tradition veut que Librada soit née au 1er siècle de notre ère à Bayona (Galice), l'antique Balcagia ${ }^{3}$. Elle aurait été la fille d'un personnage important, Lucio Catelio, roi ou consul de Lusitanie ou de Galice. C'est du moins ce que rapporte un Lectionnaire connu sous le nom de Breviario de Don Rodrigo, un personnage qui fut évêque de Sigüenza de 1192 à 1221 . En réalité ce texte est vraisemblablement l'œuvre de l'évêque Bernard d'Agen, un homme important que nous retrouverons, qui s'inspira d'un Codex plus ancien d'origine aquitaine. En témoignent les nombreuses allusions aux traditions et légendes d'Aquitaine, un pays où le culte de sainte Livrade et de sainte Quitterie - celle-ci était l'une des sours de Librada- est attesté dès le vine siècle. Le Breviario constitue en tous cas la référence hagiographique la plus ancienne que nous possédions sur santa Librada ${ }^{4}$.

La légende rapporte que la mère de Librada, la reine Calsia, avait donné le jour à neuf filles en un seul accouchement, mais qu'effrayée par cette fécondité insolite, elle ne voulut pas que l'évènement soit ébruité ${ }^{5}$. Elle appela donc sa servante, la fidèle Sila, et lui ordonna d'aller noyer secrètement les neuf enfants dans la rivière proche, près du pont de la Ramallosa, là où les eaux étaient les plus profondes. Mais la servante, horrifiée à la perspective de se rendre complice d'un tel crime, décida de conserver la vie aux petites princesses. Elle se rendit dans un faubourg de la ville habité par des chrétiens et confia les neuf sœurs à neuf nourrices, à qui elle promit une bonne indemnité. Ces femmes chrétiennes, en bonnes prosélytes, les firent baptiser et les élevèrent dans la religion chrétienne. Devenues adolescentes, les neuf princesses décidérent toutes de se vouer à Dieu.

3 Acta Sanctorum, XX juillet, "De Liberata alias Wilgeforte, virgine et martyre in variis Europæ partibus; III. "Cultus S. Liberatæ in Hipania", pp. 54-57. Henrique FLÓREZ, España Sagrada, XIV, p. 122.

4 Dr. Juan Antonio MARTínez Gómez-GoRdo, Leyendas de tres personajes de Sigüenza: Santa Librada, Doña Blanca de Borbón, Reina de Castilla y El Doncel de Sigüenza (Sigüenza, 1971), pp. 5-6.

5 Antonio CASTILlo DE LUCAS, "El parto múltiple en las leyendas y romances españoles", Retablo de tradiciones populares españoles, (Madrid: Luis Cossanas, 1963), pp. 74-88. Bartolomé PALAU, Historia de Santa Librada y sus ocho bermanas, Zaragoza, 1569. Baltazar PORRENo, Discurso de la Vida y Martirio de la gloriosa Virgen y Mártir Santa Librada, Española y Patrona de la Iglesia, y Obispado de Sigüenza (Cuenca: Salvador de Viader, 1629). 
C'est alors qu'un édit impérial ordonna la répression contre les chrétiens. Librada et ses sœurs furent traduites devant le roi Catelio qui voulut les contraindre à sacrifier aux idoles. Apprenant avec surprise qu'elles étaient ses filles, il leur fit miroiter les douceurs de la vie au palais, les exhortant, si elles souhaitaient rester vierges, à se vouer au culte de Vesta. Mais Librada répondit en leur nom qu'elles ne renieraient pas leur foi. Le roi leur proposa de réfléchir au choix qu'il leur proposait: ou elles sacrifieraient aux idoles, ou elles mourraient... Pour éviter que le roi ne devint parricide, les neuf sœurs décidèrent alors de quitter la ville secrètement. Elles n'en eurent pas le temps; on les fit prisonnières et, un 18 janvier, elles reçurent le martyre parce qu'elles étaient restées fidèles à leur foi.

On retrouve dans ces vies arrangées, où les emprunts à d'autres vies sont notoires, certains traits classiques de l'hagiographie. Ainsi en est-il du conflit entre un père demeuré farouchement païen et des filles qui se sont faites chrétiennes: tout à la fois conflit de religion et conflit de génération, où le père fait figure de symbole du passé, et les filles d'adeptes de la vraie foi tournées vers l'avenir. Résumons: nous sommes au premier siècle après J.C. et la persécution est peut-être l'œuvre de Tibère, à moins qu'il ne s'agisse de Domitien. Un roitelet lusitanien prétend faire honorer les dieux des païens à ses neuf filles, dont il a découvert sur le tard l'existence et la foi chrétienne. L'affaire prend un tour dramatique, dans la mesure où elle recouvre un enjeu de pouvoir: c'est en effet par quelqu'un de son propre sang que le potentat local est nargué. Comme il ne peut reculer sans se déconsidérer, l'inéluctable engrenage se met en place qui conduit au martyre tragique.

On sait que le part multiple appartient également à tout un vieux fond mythique de l'Europe centrale et occidentale ${ }^{6}$. Alors qu'elle est enceinte, une princesse qui fait preuve d'une grande sécheresse d'âme en refusant l'aumône à une pauvresse surchargée d'enfants en bas âge, est punie dans sa progéniture en mettant au monde neuf filles "d'une même ventrée". Redoutant d'être accusée d'adultère, parce que des couches aussi prolifiques ne pouvaient être imaginées comme résultant d'un seul embrassement, elle se résoud au pire, c'est à dire à faire disparaître tous ses nouveau-nés. Un part animal engendre un comportement animal: on noie les portées trop nombreuses de chiens et de chats...

6 L'hyperfécondité résultant de l'insulte lancée, la femme prolifique comparée à une truie, constituent des thèmes traditionnels du "romance primitivo". On se reportera à la belle étude de François DELPECH, "Como puerca en cenegal": Remarques sur quelques naissances insolites dans les légendes généalogiques ibériques", dans La condition de la mujer en la Edad Media, Coloquio Hispano-Francès, 5-7 de noviembre de 1984 (Madrid: Casa de Velázquez, 1986), pp. 343-370. 
Le part multiple, synonyme de déréglement sexuel, a nourri l'inspiration littéraire. Deux "romances" fameuses, Los siete infantes de Lara, et LoS Porceles de Murcia de Lope de Vega, ont repris ce thème des naissances multiples ${ }^{7}$. Le légendaire revient toujours sur le même thème: la mère effrayée par les conséquences de son comportement, et redoutant en particulier d'être accusée d'infidélité, tente de faire disparaitre les traces de son accouchement; mais quelqu'un veille qui empêche l'irréparable.

La légende généalogique de santa Librada et de ses sœurs laisse dans l'incertitude un point essentiel, celui du martyre. On affirme généralement que les princesses auraient été décapitées, et c'est bien ainsi que le retable de la cathédrale de Sigüenza figure leur mort; du moins celle de Librada. Mais il est vrai aussi que des textes parlent de crucifixion et que certaines images ou tableaux reprennent, à partir du xvIIe siècle, le thème de la vierge crucifiée. Ce qui témoigne de l'incertitude qui règne à propos de la vie de la sainte.

\section{UNE PIEUSE FALSIFICATION}

Au cours des siècles, le culte a traversé bien des vicissitudes; la tradition s'affaiblit à tel point qu'au Xvıe siècle on en vint à attribuer l'origine des reliques à un évêque de Sigüenza nommé Simon, qui les avait obtenues, disait-on, du pape Boniface VIII en $1300 \ldots$ La confusion en réalité était née de la translation des restes de la sainte, dont l'évêque était un grand dévot, dans une urne d'argent; cette piéce d'orfévrerie, la fameuse "arca" conservée aujourd'hui encore dans une niche du retable, avait été exécutée par des artistes florentins. Il n'en fallu pas plus pour que s'accrédite l'idée que la sainte était originaire d'Italie, et notre Librada fut confondue alors avec la santa Librada de Côme! ${ }^{8}$

Devant tant de légèreté, la Curie inquiéte réagit. Lorsqu'en 1568 un Bref de Pie V supprima les prières qui n'avaient pas été instaurées depuis plus de deux siécles ou n'avaient pas été approuvées par le Saint-Siège, on saisit le prétexte pour interdire dans le bréviaire de Sigüenza les prières consacrées à santa Librada; ce qui était une atteinte directe au culte de la sainte et un affront pour les esprits pieux du diocèse. Le chapitre et l'évêque de Sigüenza le comprirent et se mirent à rassembler toutes sortes de "preuves" pour obtenir le rétablissement des fameuses prières. Et c'est dans ce contexte que fut publié un demi siècle plus tard, en 1619,

\footnotetext{
7 Id., pp. 353-354.

8 Dr. Juan Antonio MarTínez GÓmEZ-Gordo, Op. cit., pp. 5-6.
} 
la Falso Cronicon de Flavio Dextro qui mélangeait le légendaire de santa Librada et celui de sainte Wilgeforte, la «vierge forte». L'auteur de cette pieuse malversation, écrite sous le pseudonyme de "Padre Julian", était le Père Jeronimo Roman de la Higuera, un homme à l'imagination fertile, déterminé à compléter, pour la bonne cause, certaines lacunes qui pouvaient exister dans l'hagiographie de la sainte. Il sut donner une apparence de vérité historique à un texte où le légendaire concernant la "vierge forte" et barbue de l'Europe du nord, dont le culte provenait d'une interprétation erronée du "Volto Santo" de Lucques, se mêlait habilement à des fragments de la "Vita" de santa Librada ${ }^{9}$. Il sut en particulier s'appuyer sur les annotations que le jésuite Molanus avait faites au martyrologe d'Usuard en 1563, pour présenter la nouvelle Vita de Librada comme un article de foi ${ }^{10}$.

Nouvel avatar du Christ de Lucques, Librada, avec ou sans barbe, devint un protomartyr de la Croix. A Sigüenza, on associa désormais dans la dévotion Librada et Wilgeforte. Comme en témoignent les cantiques, les gozos, et les invocations qui circulèrent sous forme de feuilles volantes. La très active "Cofradia Seguntina de santa Librada", qui venait d'être fondée, contribua alors efficacement à la diffusion du faux culte, aussi bien en Espagne -en 1682, la Sacrée Congrégation des Rites y autorisa la célébration du nouveau culte le 20 juillet ${ }^{11}$ - qu'au Portugal ou en Amérique latine.

\section{UN RETABLE, DEUX IMAGES}

Le tombeau du Doncel est sans aucun doute l'œuvre d'art la plus connue de la cathédrale de Sigüenza. Mais l'autel de Santa Librada situé dans le bras nord du transept n'en est pas moins l'un des meilleurs exemples de l'art plateresque en Espagne. Il semble bien que ce retable ma-

9 Sur Wilgeforte, Ontcommer, Kümmernis existe une abondante bibliographie. Deux études sont essentielles: G. SCHNÜRER et J. M. RITZ, "Sankt Kümmernis und Volto Santo"; Studien und Bilder; Forschungen zur Volkskunde, n. ${ }^{\circ}$ 13/15 (Düsseldorf, 1934); J. GESLER, La Vienge barbue; la légende de sainte Wilgeforte ou Ontcommer (Paris: A. Picard, 1938). Cf. aussi Jacques GÉLIS, "Wilgeforte, la sainte crucifiée, dans Populations et Cultures; études réunies en l'bonneur de François Lebrun, (Université de Rennes 2, 1989) pp. 315-323.

10 Dr. Juan Antonio MarTínez GÓMEZ-GoRDo, Op. cit., p. 7.

11 La date du martyre de la sainte, on le sait, est le 18 janvier; mais depuis 1538, c'est le 20 juillet, date de la translation des reliques dans la chapelle actuelle, qui est le jour de la fête de la sainte. 


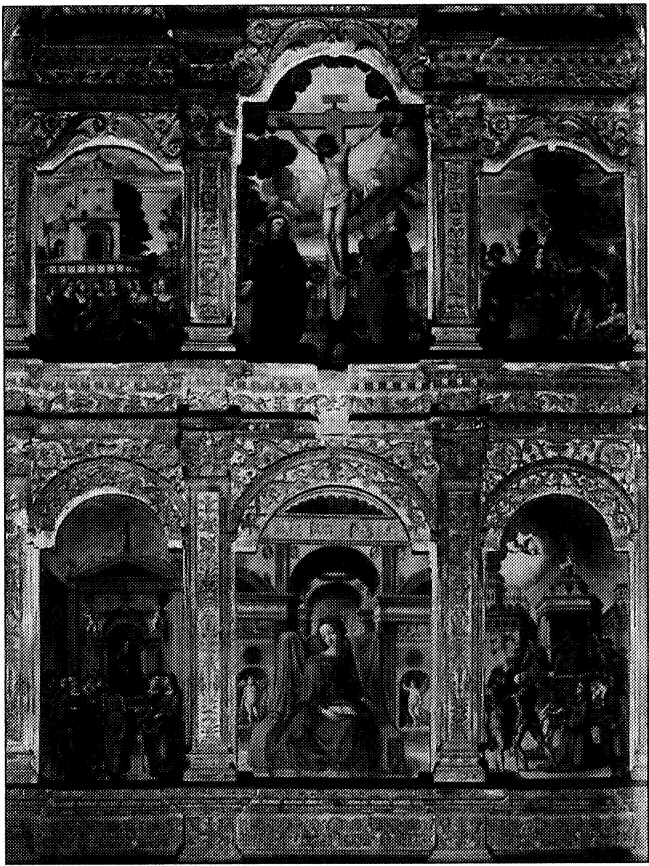

FIGURE 1.-La partie peinte du retable, attribué à Juan de Pereda, figurant sur le mur nord de la cathédrale de Sigüenza date de 1525-1526. Outre la scène très fine de la Crucifixion, devant laquelle sera placée à la fin du xvire siècle une statue de sainte Wilgeforte, et la représentation très classique de santa Librada assise sur un trône de justice, l'artiste a fait figurer quatre épisode de la vie des neuf sœurs. Ph. J.G. niériste, où transparaît l'influence italienne, celle du Perugin et surtout de Raphaël, ait été conçu par le jeune Covarrubias après 1520 . Il s'agit en réalité d'une combinaison du type retableautel-sépulture ${ }^{12}$.

L'ensemble se compose de trois registres superposés ${ }^{13}$. Le registre central, le plus important en raison de sa valeur religieuse et symbolique, comporte une cavité fermée. par une grille ouvragée: là est solennellement exposée l'urne contenant les précieuses reliques de la patronne de la cité et du diocèse. Dans des niches, situées de part et d'autres, sont figurées deux scènes majeures de la vie de la Vierge, l'Annonciation et la Visitation. Elles sont complétées au registre supérieur par une Assomption. Ces trois scènes de la vie de la Vierge sont sculptées. Le registre inférieur, celui de l'autel, comporte en sa partie centrale six scènes peintes, une Crucifixion et cinq épisodes de la vie de santa Librada, attribuées au grand artiste Juan de Pereda. Dans des niches des registres inférieur et médian figurent les statues des huit sœurs de la sainte.

12 Sur la chapelle et l'autel de santa Librada, cf. Manuel PÉreZ VILLAMIL, La catedral de Sigüenza (Madrid, 1899), p. 47 et suivantes. Antonio HERRERA CASADO, Glosario Alcarreño, t. II, Sigüenza y su tierra (Guadalajara, 1976), pp. 73-76. Aurelio de FEDERICO, La catédral de Sigüenza ("Los Monumentos Cardinales de España" (Madrid, 1954), pp. 42-58. Chandler R. POST, A History of Spanish Painting, t. IX (Cambridge Mss., 1947), pp. 697-701.

13 Description détaillée dans Elías TORMO, "El Retablo de Sigüenza de Juan de Pereda, en 1525", Boletín de la Sociedad Española de Excursiones, t. XXIV (1916) pp. 225-229. 


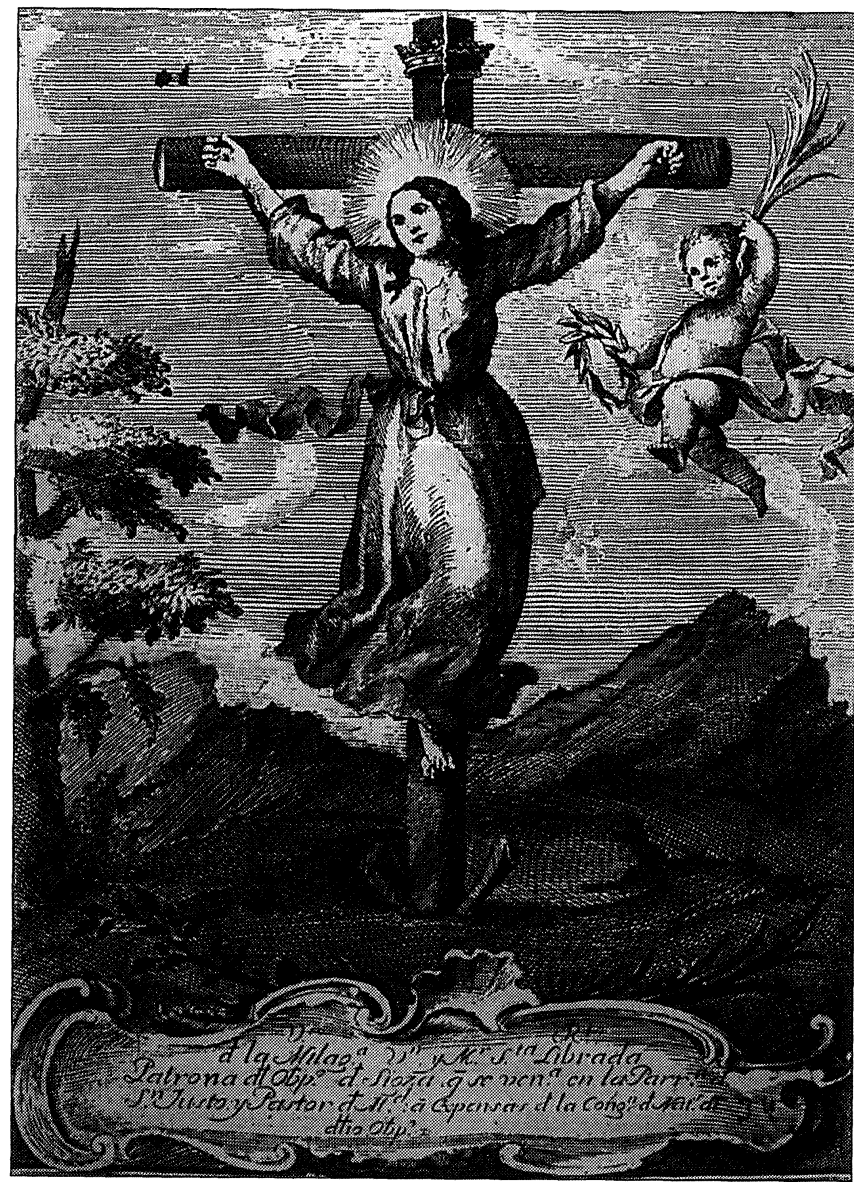

FIGURE 2.-Cette gravure de dévotion de la première moitié du Xvirie siècle a eu pour modèle la sculpture de santa Librada crucifiée qui fut installée sur le retable de la cathédrale en 1694, alors que le culte de sainte Wilgeforte se répandait. La légende indique qu'elle était vénérée en l'église de San Justo y Pastor de Madrid. Madrid Biblioteca Nacional, Dep. Bellas Artes $(19,5 \mathrm{~cm} / 14 \mathrm{~cm})$
Don Fadrique de Portugal, le prélat qui avait fait édifier ce chef d'œuvre, était un personnage important ${ }^{14}$. Fils du comte Alonso de Faro, il appartenait en effet au lignage des souverains portugais. Il avait été d'abord bé$\mathrm{n}$ é $\mathrm{d}$ i c t i $\mathrm{n}$ au monastère de Ripoll, puis chanoine d'Albarracín, avant d'être nommé évêque de $\mathrm{Ca}$ lahorra en 1503 , de Segorbe en 1508 et de Sigüenza en 1519 , 1 'année où Charles de Habsbourg, déjà roi d'Espagne, accède à l'Empire sous le nom de

Charles-Quint. C'est donc juste après sa nomination au siège de Sigüenza qu'il passe commande du retable de santa Librada et de l'autre retable disposé en angle à proximité: l'actuel "retable de Don Fadrique». Nommé par l'empereur vice-roi de Catalogne en 1525, il deviendra archevêque de

14 Augustin REDONDO, Antonio de Guevara et l'Espagne de son temps. De la carrière officielle aux cuures politico-morales (Genève: Droz, 1976) p. 406 note 317. 
Saragosse en 1532. Ce courtisan distingué n'est pas un ingrat; il participe même activement à l'enracinement du pouvoir du nouveau souverain. Et le retable consacré à la sainte patronne de la cité et du diocèse participe de cette démarche.

Les sources iconographiques représentent deux scènes différentes du martyre de santa Librada. L'une, image classique du martyre chrétien, fait mourir la sainte par décollation: la sainte rend l'âme lorsqu'un bourreau enturbanné finit par lui trancher la tête avec son cimeterre. C'est cette scène que reproduit l'un des petits tableaux composant le retable de la cathédrale de Sigüenza. Mais un changement est perceptible dans le système de représentation iconographique à partir du Xvire siècle; les images représentent désormais santa Librada crucifiée. Il s'agit d'une part, de gravures de dévotion largement diffusées au milieu du xvıIIe siècle, dans le diocèse de Sigüenza et dans celui de Madrid — par le "Novenario de la Cofradía de Madrid", par exemple- d'autre part de statues, comme celle qui fut placée en 1694 dans la partie centrale du retable de Pereda, et qui est aujourd'hui conservée dans la "Sacristía de los Mercenarios" de la cathédrale ${ }^{15}$. D'autres représentations de santa Librada crucifiée peuvent d'ailleurs être citées: la plus connue constitue l'une des belles pièces du "Museo Nacional de Escultura de Valladolid"; une sculpture datant sans doute du début du Xvile siècle, exposée au "Museo Diocesano de Sigüenza», provient d'un ancien ermitage proche de Sigüenza, la "Ermita de la Guardera"; une autre figure au grand rétable du "Monasterio de Agustinas de Vistalegre" près de Vilagarcía de Arousa (province de Pontevedra); d'autres sculptures, toutes remarquables, existent dans l'église de Bayona en Galice, l'antique Balcagia où la tradition fait naître Librada et ses sœurs, dans la cathédrale de Tuy et dans l'église d'Atienza à l'ouest de Sigüenza. Dans l'église de Pastrana, enfin -mais cette liste est loin d'être exhaustive- une petite peinture représente santa Librada sur sa croix entourée d'une magnifique guirlande de fleurs.

Le culte a été autrefois géographiquement étendu, et des images de Santa Librada crucifiée ont existé hors de la Castille et de la Galice. Ainsi, à Barcelone jusqu'en 1909, un culte était rendu à la sainte en l'église San Cucufate; et l'on sait qu'elle était également l'objet d'une dévotion très populaire en Aragón (Burbáguena) dans les Asturies (reliques fameuses à Oviedo), au Portugal, et même aux Indes (reliques dans la cathédrale de Panamá) ${ }^{16}$.

15 Cette sculpture date du début du xviıe siècle et fut sans doute l'œuvre de Giraldo de Merlo.

16 Cf. Dr Don Diego Eugenio González Chantos y Ullarai, Santa Librada, Virgen y Màrtir, Patrona de la Santa Iglesia, Ciudad y Obispado de Sigüenza (Madrid, 1806), pp. 152-158. 


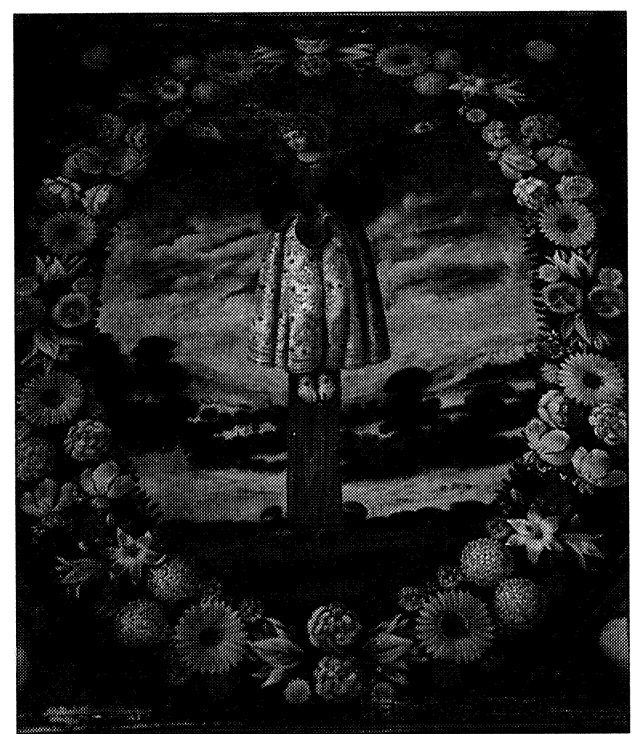

FIGURE 3.-Santa Librada est ici représentée crucifiée sur un Golgotha castillan, dans un écrin de fleurs. Peinture du xvile siècle. Chapelle de l'église paroissiale de Pastrana. Ph. J.G.

des images et sur un légendaire falsifié.

Nous savons qu'en 1694, une statue de Librada crucifiée a été placée devant le calvaire du retable de Juan de Pereda ${ }^{17}$; ce qui était une manière d'officialiser cette image de la sainte. Elle masqua alors le Christ, si bien que la Vierge et saint Jean paraissaient honorer la protectrice de la cité: un véritable détournement d'image! Malgré les vicissitudes de l'histoire, et en particulier le pillage de la cathédrale au cours de l'occupation napoléonienne, la statue demeura sur le retable jusqu'au début du xxe siècle ${ }^{18}$. Ainsi s'accrédita officiellement l'idée que santa Librada avait été victime du même supplice que le Christ: Pouvait-on souhaiter martyre plus exemplaire, et patronne plus estimable?

17 Dr Juan Antonio Martínez Gómez-Gordo, Op. cit., p. 21.

18 "Tabla central alta: el Calvario. La postiza Santa Librada, de escultura del siglo XVII (crucificada, según el error nuevo de entonces) oculta la tabla, pero se acierta a ver que las figuras de la Virgen María y el evangelista San Juan son muy bellas”. Elías TORMO, "El retablo de Sigüenza de Juan de Pereda, en 1525", Boletín de la Sociedad Española de Excursiones, t. XXIV, 1916, p. 227. 


\section{LIBÉRÉE ET FORTE}

Le choix par Bernard d'Agen de santa Librada comme symbole d'une ville libérée - Sigüenza librada - est clair. En amenant d'Aquitaine les reliques d'une sainte que sans doute il honorait auparavant, il contribuait à jeter les bases d'un véritable culte identitaire dans la cité reconquise de haute lutte sur les Maures, le jour de la Saint Vincent 1124. Les habitants de la ville et du diocèse allaient se reconnaître désormais dans cette sainte, dont le nom même rappelait la liberté retrouvée. La crypte de la cathédrale que l'on commença immédiatement à édifier au cour de la cité reçut les reliques. Et pour mieux les honorer, quatre siècles plus tard, Don Fadrique fit installer les précieux restes dans le bras nord du transept, que peu de temps auparavant l'un de ses prédécesseurs, l'évêque Mendoza, avait entrepris de décorer.

Le thème de la vierge forte, patronne et protectrice de la cité, a également trouvé à Sigüenza un terrain favorable. L'image d'une vierge armée et casquée, incarnation de la ville dont elle est le palladium est ancienne et se retrouve en bien des lieux. La "mujer fuerte", ou la "virgen fuerte", est en effet un thème récurrent de la tradition biblique et antique, auquel la Renaissance va donner un nouveau souffle. Ainsi, au début du XVIe siècle, à Florence, Fra Bartolomeo représente-t-il une Minerve-Pallas casquée, lance et bouclier en main ${ }^{19}$.

On retrouve cette symbolique, sous une forme cependant moins belliqueuse, dans la peinture et la statuaire aux Xvire et XVIIIe siècles. Dans la chapelle de la Vierge —Camarín de Nuestra Señora" du monastère de Guadalupe, les sculptures des huit femmes fortes de l'Ancien Testament - Marie la prophétesse, Déborah, Jael, Sara, Ruth, Abigail, Esther et Judithont été installées en 1736 dans les niches ménagées dans les piliers.

La "mujer fuerte" de la tradition chrétienne n'a pas besoin, elle non plus, de l'accoutrement guerrier pour jouer son rôle de protectrice d'un pays et d'une cité. Sa force n'est pas physique mais essentiellement morale, spirituelle; c'est sa force d'âme, la solidité de sa foi qui en fait une femme exemplaire, digne de la confiance de ses protégés. Dans le retable de Don Fadrique, c'est bien ainsi que Librada est représentée. Comme le veut la tradition chrétienne, elle porte la palme du martyre et a sur les genoux, grand ouvert, le livre de sagesse. Elle siège, les yeux baissés, avec

19 Le tableau de 1506 a figuré à l'exposition Le Xve siècle florentin au Louvre; catalogue d'exposition (Paris, 1982), pp. 9-10. Sur le thème de la "femme forte", cf. Sara F. MATTHEWS-GRIECO, Mythes et iconographie de la femme au xve siècle français; images d'un univers mental, Thèse EHESS (Paris, 1982), pp. 145-148. 


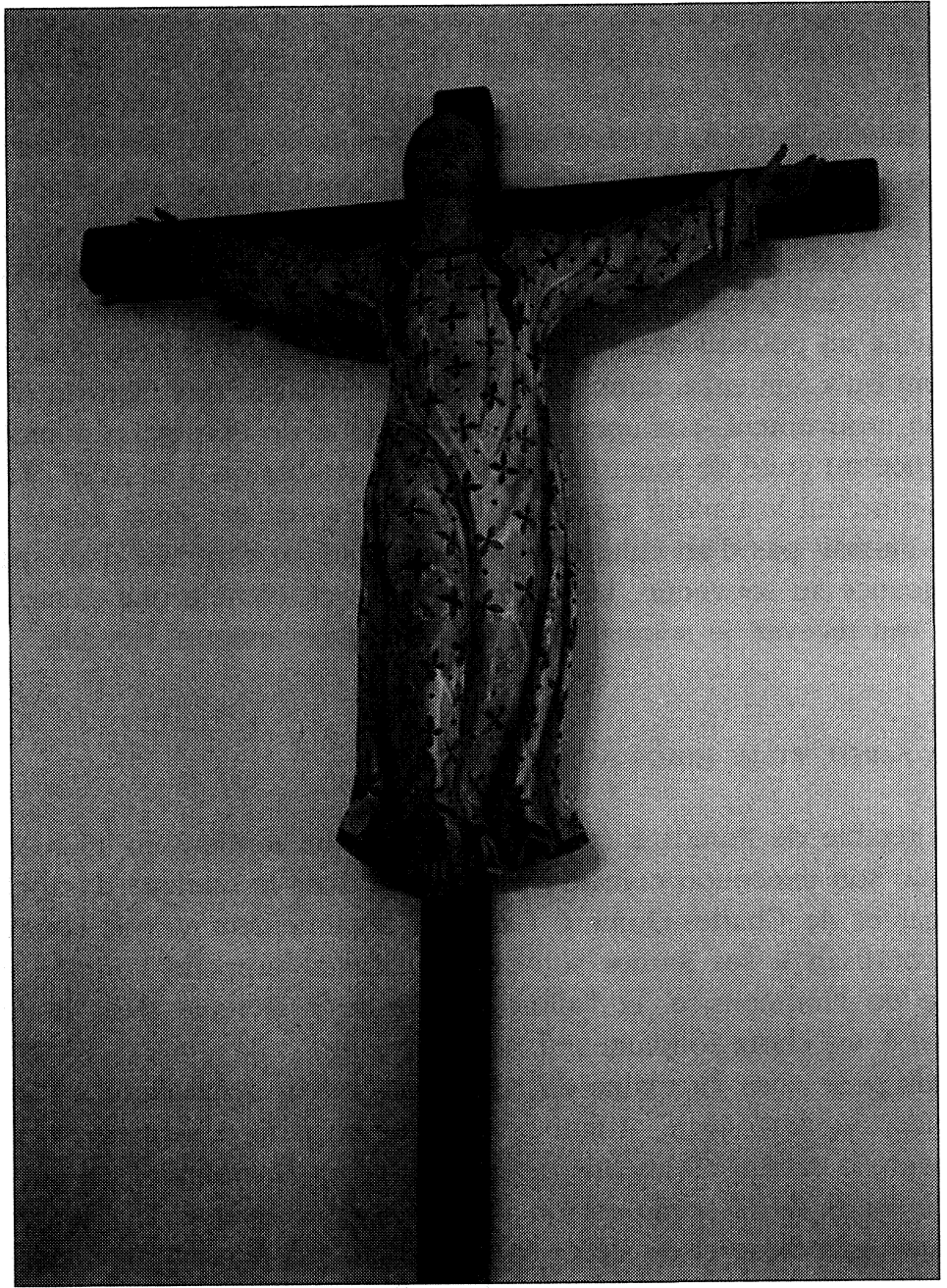

FIGURE 4.-Cette sculpture aujourd'hui au "Museo Diocesano" de Sigüenza provient de la "Ermita de la Guardera", dans les environs de la ville, où elle était déjà honorée en 1620. C'est sans doute dans cet ermitage, au lieu-dit "La Santa", que s'enracina le culte de santa Librada crucifiée. Ph. J.G.

la gravité et l'humilité qui sied à une sainte, sur un trône de majesté recouvert d'une tenture semblable à une peau trop grande...

Il est possible que le changement d'image - le passage de la Vierge décapitée à la vierge crucifiée- ait été d'abord expérimenté en un lieu isolé, extérieur à la ville, avant d'être agréé dans la cathédrale. Près de Sigüenza, sur les hauteurs de Pelegrina, au lieu-dit "La Santa", existait aux siècles passés 
un vieil ermitage, "la Ermita de La Guardera", dont ne subsiste plus que la base des murs ${ }^{20}$. Une image de santa Librada crucifiée y était vénérée vers 1620 , sans que nous sachions quand et par qui le culte avait été fondé, et quels étaient les liens existant entre cet ermitage et la ville de Sigüenza. S'agissait-il d'une tentative comparable à "l'invention" de san Frutos à Ségovie en 1518, ou de san Segundo à Ávila en $1519^{21}$ ? Dans les deux cas, c'était le contexte politique du moment qui avait conduit à l'émergence d'une dévotion locale: le saint patron allait incarner l'esprit de résistance de la ville au pouvoir absolutiste du monarque. Or, c'est précisément à ce moment qu'à Sigüenza, dans une cité moins engagée que Ségovie et Ávila dans le mouvement "comuneros", Don Fadrique de Portugal a contribué à la relance du culte de santa Librada, dont le retable qu'il lui avait consacré est aujourd'hui le témoin privilégié. A la différence des deux autres villes, il ne s'agissait pas d'un culte contestataire, mais au contraire d'un symbole d'allégeance au souverain. Librada y apparaissait comme une sainte légitimiste, qui recevait le soutien inattendu du héros antique: Hercule.

\section{LA SAINTE ET LE HÉROS ANTIQUE}

Le retable de Pereda a été vraisemblabement terminé en 1526; c'est à dire que son exécution coïncide exactement avec le mariage d'Isabelle de Portugal et de Charles-Quint ${ }^{22}$. On peut légitimement y voir l'hommage d'un courtisan à ses jeunes souverains: Librada rappelle l'origine lusitanienne de l'impératrice, et l'allusion discréte à Hercule dans le retable renvoie à un choix fondamental de l'empereur.

Mais pour saisir l'actualité du retable, sa valeur symbolique en ce premier tiers du Xviè siècle, il faut revenir sur la partie essentielle, celle qui représente la sainte au-dessous de la Crucifixion. Le trompe-l'œil qui constitue le fonds de la scène est on ne peut plus classique: il s'agit de l'architecture grandiose et du décor solennel d'un palais royal à l'antique, tels qu'on l'imaginait en cette période de la Renaissance.

20 Dr Juan Antonio MARTínez GÓMEZ-GORDO, Op. cit., p. 16.

21 Sur le rôle complexe des patronages locaux dans la lutte politique qui opposent les villes de Vieille Castille au pouvoir monarchique, signalons l'excellente étude de María CÁTEDRA, "L'invention d'un saint. Symbolisme et pouvoir en Castille», dans Terrain, carnets du patrimoine etbnologique, n. ${ }^{\circ} 24$, "La fabrication des saints" (mars 1995), pp. 15-32.

22 La famille de Fadrique de Portugal est liée à la maison d'Espagne; sa mère, une comtesse portugaise, est marraine de l'union de Charles-Quint et Isabelle de Portugal en 1526. Je dois ce renseignement, ainsi que plusieurs références bibliographiques, à Jean-Marc DEPLUVREZ. 
Certains détails auxquels il faut prêter attention ne manquent pas de surprendre dans une composition tout à la gloire d'une sainte patronne. Une partie du décor est en effet éminemment païen, puisqu'il illustre les travaux d'Hercule. Mais que vient donc faire dans un retable chrétien ce héros de la mythologie gréco-romaine, dont on a pu dire qu'il était "le plus humain des dieux ${ }^{23}$ ? Le lien est plus cohérent qu'il n'y paraît à première vue. Tradition classique et tradition chrétienne viennent en réalité contribuer ici à l'exaltation de l'idée impériale.

Hercule occupe en effet une place importante dans la définition de l'image mythico-hérö̈que de Charles-Quint ${ }^{24}$. Pourtant jusqu'au milieu du xve siècle, l'exaltation de la monarchie à travers les personnages de la mythologie ou de l'histoire romaine n'était pas très bien vue en Espagne. Certes, la figure d'Hercule n'avait pas été totalement oubliée pendant la période médiévale, mais elle avait été éclipsée au profit des héros de la tradition locale, comme le Cid par exemple. Or, elle trouve à partir des années 1520 une dimension, une signification nouvelle ${ }^{25}$. Toute une littérature s'intéresse à ce modèle de vie exceptionnel, à ce héros de l'Antiquité, en qui l'on voit le précurseur de Samson et David, ou la préfiguration païenne du Messie.

Un ouvrage écrit par le marquis de Villena au milieu du Xve siècle et publié en 1483, Los Doce trabajos de Hércules, constitue un bon exemple de cette utilisation de la mythologie antique à des fins morales et politiques ${ }^{26}$. Ce que l'auteur retient surtout du héros légendaire, c'est la valeur chevaleresque de sa figure. Hercule est un chevalier sans peur et sans reproche, protecteur des pauvres, des femmes et des invalides. Et Villena donne alors une minutieuse interprétation allégorique des douze travaux;

23 Marc-André JUNG, Hercule dans la littérature française du xve siècle. De l'Hercule Courtois à l'Hercule Baroque, (Genève: Droz, Travaux d'Humanisme et Renaissance, 1966), p. 6.

24 Robert B. TATE, "Mythology in Spanish Historiography of the Middel Ages and the Renaissance, , Hispanic Review, t. XXII (1954), pp. 1-18. José Antonio Maravall, Carlos Vy el pensamiento político del Renacimiento (Madrid, 1960). Fernando CHECA CREMADES, Carlos $V y$ la imagen del héroe en el Renacimiento (Madrid, Taurus, 1987).

${ }_{25}$ Cf. l'éclairage et les nombreuses références bibliographique que donne JeanMarc DEPLUVREZ dans «Hercule à Tolède. Du palais enchanté aux réjouissances urbaines", La légende (Madrid, Casa de Velazquez/ Editorial Universidad Complutense, 1989) pp. 155-172.

26 E DE VILLENA, Los doce trabajos de Hércules, publié par Margarita MONREALE, série II de la Biblioteca Selecta de Clásicos Españoles, vol. XX (Madrid, 1958). Sur le thème des modèles de vie à imiter au début du xvie siècle, cf. Juliana SÁNCHEZ AMORES, aSeguimiento de la "Psicomaquia" a través de los tratadistas desde el mundo medieval al moderno", Archivo Español de Arte, 244 (1988) pp. 430-440. 


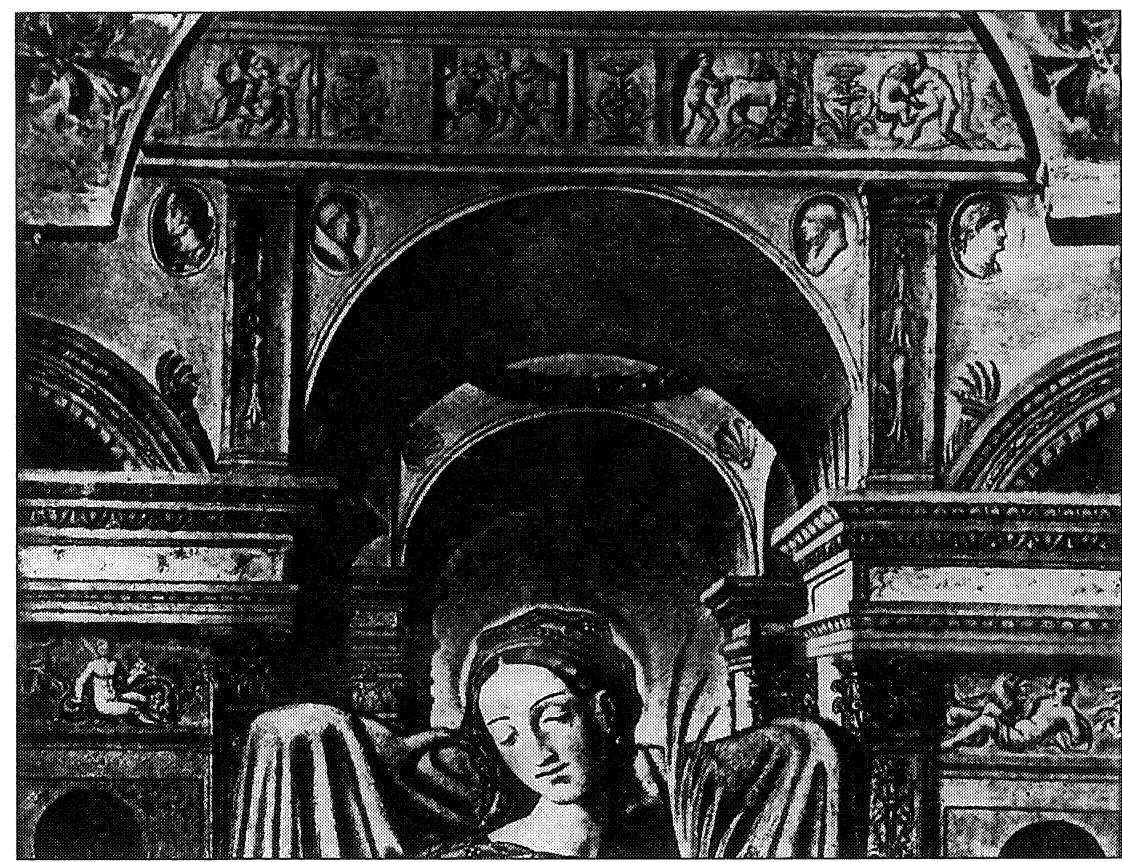

FIGURE 5.-Sur le linteau situé au-dessus de santa Librada figurent quatre des douze travaux d'Hercule. Ces scènes en trompe-l'œil sont très semblables aux sculptures de la façade de l'église San Salvador d'Úbeda, construite sur la commande des Cobos. Dans le cartouche à gauche de la sainte, Hercule enfant étouffe les serpents. Ph. J.G.

chacun renvoie à l'un des "états" de la société: prince, prélat, marchand, travailleur....

Mais le héros légendaire nourrit aussi l'idéologie impériale, dont il devient l'une des références privilégiées. Parce qu'il établit un passage entre le monde des hommes et celui des dieux, Hercule contribue à l'exaltation du monarque absolu, qui prétend détenir son pouvoir de Dieu seul. Ce que l'on glorifie maintenant à travers le personnage d'Hercule, c'est la force, la gloire, du prince. En montrant de manière didactique le triomphe des vertus sur les vices, l'œuvre de Villena fournit les bases de référence du nouvel "imperium". Nouvel Hercule, le souverain exalte les vertus et purge ses terres des vices qui l'infectent.

L'allusion discrète - le lieu l'impose- à Hercule dans le retable de Sigüenza constitue sans doute l'un des tous premiers exemples d'un genre pictural appelé à une grande fortune en Espagne ${ }^{27}$. Désormais, les palais

27 C'est en 1522, donc trois ans avant que Juan de Pereda exécute le retable de Santa Librada, qu'AlCIAT publie la première édition de son ouvrage Les Emblêmes, où 
vont se couvrir d'œuvres peintes destinées à chanter les exploits guerriers du héros, devenu l'ancêtre mythique du prince ${ }^{28}$. Hercule est le représentant du Bien et le triomphateur des vices, comme Charles-Quint est le défenseur des vertus chrétiennes contre tous les ennemis de la Religion et de l'Etat.

Dès lors, on comprend mieux la signification du message livré par le retable de Sigüenza. Don Fadrique, en évêque-courtisan, contribue à l'élaboration d'une mythologie impériale, à la définition de l'simperium" du jeune souverain. Les scènes évoquant les travaux du héros gréco-romain ont été choisies avec discernement. C'est principalement la frise disposée au-dessus de santa Librada qui va retenir notre attention ${ }^{29}$. Cette frise est supportée par les deux colonnes qui encadrent la sainte assise sur son trône. Quatre scènes relatent les travaux. La première, à gauche, figure le combat contre les Centaures, qui passent pour être le symbole des comportements malfaisants et criminels. L'enseignement de cette fable est clair: Pour le repos des peuples, la Justice est la vertu principale qui doit dominer la conduite du Prince. La Justice d'une part, l'esprit chevaleresque de l'autre; c'est ce que veut signifier précisément le sujet de la quatrième scène, la lutte contre le Lion de Némée: l'allégorie guerrière associée au thème du chevalier redresseur de torts, vainqueur des ennemis de la patrie.

Don Fadrique s'appuie en effet sur le culte identitaire à santa Librada pour affirmer le pouvoir de son souverain. C'est en faisant référence au héros de l'Antiquité devenu le symbole de Charles-Quint qu'il prétend honorer le sainte de Sigüenza. Cette recharge du culte prend alors une signification politique d'autant plus évidente que le prince vient de triompher de ses ennemis intérieurs et extérieurs. Voyons cela de plus près.

Au-dessous de la frise, dans deux cartouches, on a représenté Hercule dans la première étape de son cycle de vie: dès son berceau, il étouffe les serpents, comme le jeune Charles-Quint, dès le début de son règne, vient d'étouffer les serpents de la discorde en Europe et en Espagne. Nous sommes en effet en 1525; François Ier, le grand rival, vient d'être vaincu et fait prisonnier à Pavie; quelques années auparavant, Charles-Quint a effectué un premier séjour dans la péninsule, puis a fait réprimer durement la révolte des "Comunidades" et des "Germanías" qui menaçait son

les allégories sont personnifiées en scènes hérö̈ques. Il n'est pas impossible que Pereda l'ait connu et s'en soit inspiré.

28 Fernando CHECA CREMAdes, Pintura y escultura del Renacimiento en España (Madrid, 1983), p. 200.

29 Cette analyse s'inspire de l'étude de Antonio HERrera CASADO, Sigüenza y su tierra..., en particulier le chapitre "Hércules en Sigüenza», pp. 77-80. 


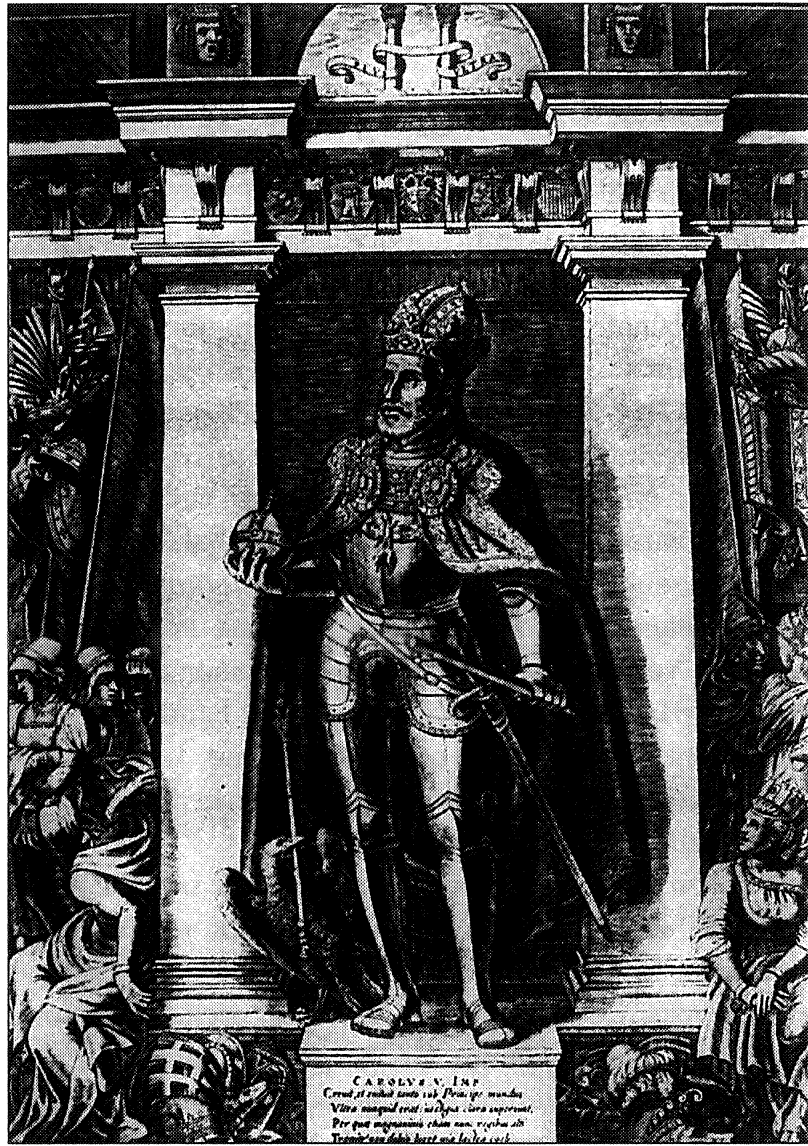

FIGURE 6.-Sur cette gravure de Charles-Quint en majesté portant tous les attributs du pouvoir impérial, il n'y a pas d'allusion directe aux travaux d'Hercule; mais dans l'ouverture au-dessus de l'entablement figure l'emblème du monarque les deux colonnes d'Hercule et la devise "Plus Ultra". Paris Bibliothèque Nationale, Estampes. autorité ${ }^{30}$. Ce sont donc les troubles politiques suscités par les "comuneros" et la victoire de la Justice souveraine sur les forces du Mal, qui sont ici figurés sous forme allégorique. Mais il y a plus: les deux scènes centrales prétendent légitimer le règne de Charles, en l'enracinant dans le passé espagnol.

Le thème est riche, sans être nouveau au xvie siècle, qui fait de l' Hercules hispanicus" le premier roi d'Espagne ${ }^{31}$, le pacificateur du peuple espagnol, celui qui l'initia à l'agriculture, fonda de nombreuses villes, comme Toledo, Mérida, Segovia, Sagunto,

30 Joseph PÉREZ, La révolution des "Comunidades" de Castille (1520-1521) (Bordeaux: Institut d'Etudes Ibériques de l'Université de Bordeaux, 1970). Edition espagnole: La revolución de las Comunidades de Castilla (1520-1521) (Madrid, Siglo XXI, 1977).

31 Parmi les ouvrages qui font d'Hercule le premier roi d'Espagne, citons celui de Florian de OCAMPO, Cronicón General de España, publié en 1543, où les trois premiers chapitres sont consacrés au séjour du héros en Espagne et en particulier aux fondations de villes. Sur le cas de Tolède, cf. Jean-Marc Depluvrez, Hercule à Tolède... Op. cit. 
Urgel, Vich, dressa à Cádiz les deux colonnes qui marquaient la fin du monde connu, éleva la fameuse tour qui porte son nom à La Coruña, et joua un rôle essentiel dans la création de Tarazona. Ce sont précisément, ces deux derniers lieux qui sont évoqués dans les scènes médianes du linteau de Sigüenza: Le vol des bœufs de Gerion, sensé se dérouler à Eritrea, "à l'occident de la Grèce", que l'on situe à l'extrêmité occidentale de l'Espagne, en Galice, et le vol dont se rendit coupable le géant Caco, alors qu'Hercule dormait; or, les auteurs espagnols situent cette scène en un lieu qu'ils appellent "las faldas del Moncayo", dont le nom viendrait de "Monte de Caco", près de Tarazona.

Ce qui se cache derrière ces représentations, c'est donc bien une entreprise de légitimation de Charles comme souverain espagnol et comme empereur. Or, les empereurs romains originaires de la péninsule, dont se réclameront les Habsbourg, ne manquaient pas de faire état des tribulations d'Hercule en Espagne. Le grand Trajan ne s'était-il pas rendu, comme Hercule, d'Espagne à Rome? Les Antonins faisaient figurer Hercule sur leurs monnaies. Quant à l'empereur Commode n'avait-il pas franchi le pas? Sa folie meurtrière ne lui permettant plus de distinguer le réel de l'imaginaire, il voulut que, revêtu d'une peau de lion de Némée, on le prît vraiment pour Hercule... Mais après tout, l'ample manteau impérial que porte Charles-Quint en son portrait officiel n'évoque-t-il pas la peau du lion de Némée jetée sur les épaules d'Hercule? Pourtant s'il faut s'attacher aux signes, ce sont bien les deux colonnes emblématiques qu'il faut retenir.

Les "colonnes d'Hercule" sont le symbole d'un empire qui vient brusquement de se dilater, puisqu'il englobe maintenant le Nouveau Monde; elles ouvrent sur un espace plus vaste que celui de l'empire romain, et à ce monde nouveau correspond un nouvel empereur. Les deux colonnes de Cádiz, et la devise "Plus Ultra" 32 , sont les emblêmes de Charles-Quint empereur; et ce sont ces deux colonnes qui supportent la frise du retable de Sigüenza.

A l'évidence, le courtisan de haut vol qu'est l'évêque Fadrique de Portugal a souhaité associer Hercule et Librada à Sigüenza, pour glorifier son souverain à travers ces deux fondateurs de villes. Avec la volonté de placer le nouvel empereur, dont les débuts en Espagne avaient été si difficiles, sous cette double protection mythique et religieuse. Si la réfé-

32 Sur la devise "Plus Ultra", cf. Marcel Bataillon, "Plus Oultre, la cour découvre le nouveau monden, dans Fêtes et cérémonies au temps de Charles-Quint, édité par Jean JaCQuOT (Paris, 1960), pp. 13-27. Frances A. YATES, "Charles-Quint et l'idée de l'Empire», dans Astrée, le symbolisme impérial au Xve siècle (Paris, Belin, 1989), pp. 17-56. E. ROSENTHAL, "Plus Ultra, Non Plus Ultra", and the column device of the Emperor Charles V", Journal of theWarburg and Courtaulds Institute, XXXIV (1971), pp. 204-228. 
rence à Librada n'a jamais été que régionale —et elle s'est d'ailleurs progressivement affaiblie, puisque le culte de la Vierge l'a emporté de plus en plus à partir du xviIIe siècle sur celui de la sainte- la référence à Hercule, elle, a été soigneusement entretenue au plus haut niveau, celui du pouvoir monarchique. En témoigne, le cycle des travaux d'Hercule réalisé par Zurbarán pour le Buen Retiro de Madrid qui, au siècle suivant, servit de référence aux grandes scènes de batailles gagnées par les souverains espagnols du Siècle d'Or ${ }^{33}$.

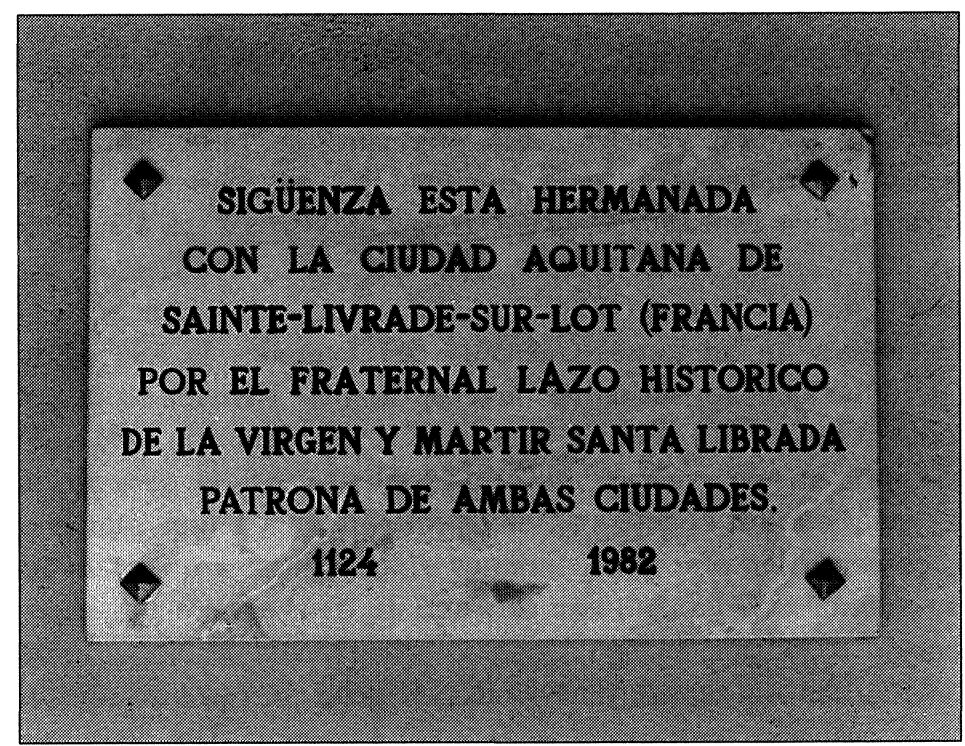

FigURE 7.-Aujourd'hui, alors que l'Eglise a pris ses distances avec le culte de Santa Librada, ce sont les autorités municipales de Sigüenza qui entretiennent le souvenir de la protectrice de la cité, grâce au jumelage avec Sainte-Livrade-sur-Lot, dont la sainte est également patronne. Plaque figurant dans le patio de la mairie de Sigüenza. Ph. J.G.

A Sigüenza, depuis 1962, santa Librada a été officiellement détrônée par la Vierge de l'Assomption, devenue patronne officielle de la ville et du diocèse. Sans doute faut-il considérer ce changement comme le point d'aboutissement d'une évolution séculaire qui, partout, a placé la Vierge

33 Jonathan BROWN et John ELLIOTT, Un palacio para el rey. El Buen Retiro y la corte de Felipe IV (Madrid, 1985). Zurbarán, catalogue d'exposition (Paris, 1988), pp. 183-185. R. LÓPEZ TORRIJOS, La mitología en la pintura española del siglo de oro (Madrid, 1985), p. 157. 
bien au-dessus de tous les saints locaux. Symbole d'universalité, la Mère du Christ s'est vue reconnaître un rôle de protectrice éminente.

Elle l'emporte, à Sigüenza, sur une "vierge forte" qui incarnait auparavant les intérêts symboliques de la cité et de la région. Ici, les circonstances politiques, et en particulier l'affirmation de l'absolutisme monarchique dans la première moitié du xvie siècle, n'ont pas peu contribué à cette évolution. La "vierge forte", dont le reliques avait servi à la refondation chrétienne de Sigüenza, avait exprimé pendant des siècles le sentiment d'appartenance des habitants de ces confins de la Vieille Castille et de l'Aragón à une terre, à un pays. Renforcée dans son rôle protecteur et identitaire par l'évêque Don Fadrique en un temps particulièrement critique pour la monarchie, elle avait été alors enrôlée dans la défense d'une idéologie impériale qui entendait valoriser le pouvoir du prince sur les pouvoirs locaux. Et, à ce titre, elle a contribué à l'intégration de cette région à l'Etat espagnol moderne.

Aujourd'hui, alors que l'Etat, l'Eglise et la société ont profondément changé, le culte de santa Librada n'est plus qu'un culte-relique dont la signification profonde est de moins en moins bien perçue. Une trace laissée par l'histoire des représentations symboliques.

JACQUES GÉLIS

Université de Paris

Reconquistada Sigüenza en 1124, Santa Librada se constituye en patrona de la ciudad. La leyenda la hace renacer de un parto múltiple y morir con sus ocho hermanas, por voluntad de su padre, por ser cristiana. Dos son las iconografías existentes de su martirio, una es la de "virgen fuerte" crucificada, la otra con la palma; y un retablo plateresco, donación de un prelado próximo a Carlos $\mathrm{V}$, celebra su memoria en la catedral seguntina. Detrás de estas representaciones, se adivina la compleja relación entre la protectora, los habitantes de la ciudad, la Iglesia y, a partir de comienzos del siglo XvI, el Estado. Santa Librada fue entonces relacionada con Hércules, el héroe predilecto de Carlos V, en defensa de los intereses políticos del soberano.

After the reconquest of Sigüenza in 1124, St. Librada became the town's patron saint. Legend has it that she was born out of a multiple delivery and that, because of her christian faith, her father had her die together with her eight sisters. On the iconography of her martyrdom, one of the representations is that of the crucified "strong virgin"; the other, more conventional, is on a plateresque altarpiece which honors her memory in Sigüenza's cathedral, a donation by a bishop close to Charles V. Behind these representations lies the complex relationship among the saint, the town's inhabitants, the church and, beginning from the 16th century, the state. St. Librada was then associated with Hercules, Charles V's favorite hero, in the defense of the sovereign's political interests. 